\title{
Oxidative stress and nitration in neurodegeneration: Cause, effect, or association?
}

\author{
Harry Ischiropoulos ${ }^{1}$ and Joseph S. Beckman ${ }^{2}$ \\ ${ }^{1}$ The Joseph Stokes Jr. Research Institute, and Departments of Pediatrics, Biochemistry, and Biophysics, Children's Hospital of Philadelphia; \\ and The University of Pennsylvania, School of Medicine; Philadelphia, Pennsylvania, USA \\ ${ }^{2}$ Linus Pauling Institute, Environmental Health Sciences Center and Departments of Biochemistry and Biophysics, Oregon State University, \\ Corvallis, Oregon, USA
}

J. Clin. Invest. 111:163-169 (2003). doi:10.1172/JCI200317638.

Oxidation and nitration of proteins, DNA, and lipids are markers of neurodegeneration in postmortem tissues. It is impossible to determine with certainty using postmortem analysis, whether oxidative stress has a primary role in neurodegeneration or is a secondary end-stage epiphenomenon. Growing evidence suggests that the generation of oxidants does not result simply from an accidental disruption of aerobic metabolism, but rather from an active process crucial for the nonspecific immune defenses of the brain. While essential for survival, these processes may be inappropriately activated to cause neurodegeneration. Neurons are highly susceptible to oxidative stress, which can induce both neuronal necrosis and apoptosis. Oxidants may also have more subtle roles in compromising the integrity of the bloodbrain barrier and in producing reactive changes in astrocytes that further propagate injury. Moreover, oxidative stress appears to provide a critical link between environmental factors, such as exposure to pesticides, herbicides, and heavy metals, and endogenous and genetic risk factors in the pathogenic mechanisms of neurodegeneration, particularly in Parkinson disease. Here, we discuss some recent insights into the diverse roles and controversies about the role of oxidants in neurodegeneration. A better understanding of the role of oxidants in neurodegeneration still holds a largely unfulfilled potential to reduce the burden of both acute and chronic neurodegeneration.

\footnotetext{
Address correspondence to: Harry Ischiropoulos, Stokes Research Institute, Children's Hospital of Philadelphia, 416D Abramson Center, 3517 Civic Center Boulevard, Philadelphia, Pennsylvania 19104-4318, USA. Phone: (215) 590-5320; Fax: (215) 590-4267; E-mail: ischirop@mail.med.upenn.edu. Conflict of interest: The authors have declared that no conflict of interest exists.

Nonstandard abbreviations used: amyotrophic lateral sclerosis (ALS); manganese superoxide dismutase (Mn-SOD); $\mathrm{Cu} / \mathrm{Zn}$ superoxide dismutase $(\mathrm{Cu} / \mathrm{Zn}-\mathrm{SOD})$; hypochlorous acid $(\mathrm{HOCl})$; nitric oxide synthase (NOS); 1-methyl-4-phenyl-1,2,3,6tetrahydropyridine (MPTP); copper chaperone for $\mathrm{Cu} / \mathrm{Zn}$ superoxide dismutase (CCS).
}

\section{Oxidative processes in neuronal systems}

Advances in understanding the chemical nature of oxidative attack on biological molecules have identified many new markers with which to examine postmortem tissues for evidence of oxidative injury. These markers include protein nitrotyrosine, carbonyls in proteins, fatty acid oxidation products, and oxidized DNA bases (1-6). Although biological oxidants have traditionally been viewed as highly reactive and destructive, they can be surprisingly selective and preferentially attack specific sites on macromolecules. For example, specific mAb's raised to specific sequences from $\alpha$-synuclein containing nitrotyrosine have revealed that nitrated $\alpha$-synuclein selectively accumulates in Lewy bodies and protein inclusions in a wide range of pathologies (4) (Figure 1). Tyrosine nitration is one of the earliest markers found in Alzheimer disease brains, in the plaques of multiple sclerosis brains, and in degenerating upper and lower motor neurons in amyotrophic lateral sclerosis (ALS) patients $(2,5,6)$. The association of specific oxidative damage with sites of injury in many different types of neurodegeneration suggests a common underlying mechanism. However, these markers could simply reflect secondary epiphenomena rather than having a causal role. A clear delineation of the causal connections cannot be given at present, but a growing body of evidence indicates that oxidants induce distinct pathological consequences that greatly amplify and propagate injury that leads to irreversible degeneration.

When the generation of oxidants exceeds the rate at which endogenous antioxidant defenses can scavenge oxidants, proteins, lipids, DNA, and other macromolecules become targets for oxidative modification, which leads to deterioration of cellular structural architecture and signaling and ultimately death. Therefore, surviving neurons in human neurological disorders without evidence of biological oxidation could be the cells with the most effective antioxidant capacity. For example, neurons surviving in Huntington disease brains have strongly induced expression of the potent antioxidant enzyme mitochondrial manganese superoxide dismutase (Mn-SOD) (7). However, oxidative damage of biological targets does not necessarily translate to a pathogenic 


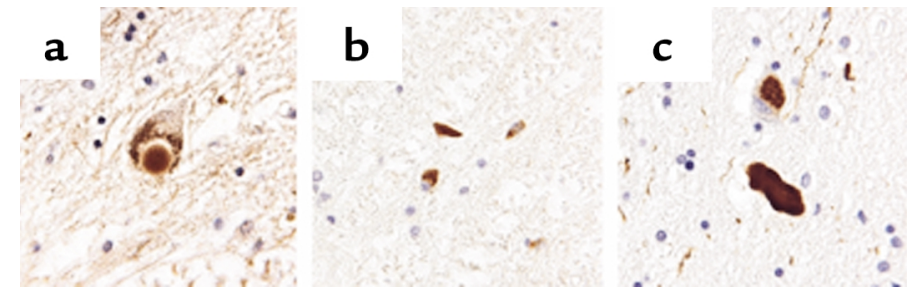

Figure 1

Specific antibodies against nitrated $\alpha$-synuclein recognize synuclein inclusions. Immunostaining with the mAb nSyn24, a nitrated $\alpha$-synuclein-specific antibody, reveals staining in nigral Lewy bodies of Parkinson disease and dementia with Lewy bodies (a), glial cytoplasmic inclusions of multiple system atrophy (b), and the Lewy body-like inclusions, neuroaxonal spheroids, and dystrophic neurites of neurodegeneration with brain iron accumulation type 1 (c). The image was provided by John Duda and Benoit Giasson (Center for Neurodegenerative Disease Research, University of Pennsylvania, Philadelphia, USA).

phenotype, because a multitude of repair processes can be activated to sustain physiological function. In keeping with this, succumbing neurons may be the least proficient in repair capacity. Consistent with this view is the demonstration of decreased repair activity of methionine sulfoxide reductase, an enzymatic activity essential for repair of oxidized methionine residues, in Alzheimer disease brains (8). Methionine sulfoxide reductase (9) or oxidized DNA-repair enzymes may regulate the lifespan of mammals, as mice with mutations in the XPD gene that encodes for a DNA helicase, which is involved in both repair of oxidized DNA lesions and transcription, show evidence of premature aging (10).

Experimentally, the importance of the balance between oxidants and antioxidants has been primarily tested by two approaches in animal and cellular model systems: the genetic elimination of an antioxidant defense mechanism, and the augmentation of antioxidant defenses. In the first paradigm, downregulation of $\mathrm{Cu} / \mathrm{Zn}$ superoxide dismutase $(\mathrm{Cu} / \mathrm{Zn}-\mathrm{SOD})$ in mice and cells is associated with increased neuronal injury and death $(11,12)$. More serious consequences arise from the elimination of the mitochondrial Mn-SOD, which is generally lethal in the neonatal period (13). In addition to causing cardiac failure, the mitochondrial SOD knockout mouse suffers CNS pathology that includes mitochondrial vacuolization and oxidized lipid deposits (13). Mice deficient in glutathione peroxidase, an enzymatic pathway largely responsible for the elimination of hydrogen peroxide and fatty acid peroxides, are more sensitive to ischemia/reperfusion injury and neurotoxins (14). Recently, mice deficient in the $\alpha$-tocopherol (i.e., vitamin E) transport protein were shown to develop a delayed-onset ataxia and neurodegeneration (15). Conversely, intake of vitamin $\mathrm{E}$ has been found to retard the clinical progression of Alzheimer disease (16), and to offer small but significant benefit in ALS patients taking riluzole (17). Recently, treatment with dehydroascorbate has been shown to be protective against stroke (18). Dehydroascorbate was used because it is rapidly taken up into the brain and is reduced to ascorbate. Deficiency in the ascorbate transporter in mice causes lethal cerebral hemorrhages shortly after birth (19).

Metals such as copper and zinc have been shown to accelerate amyloid deposition (20). Redox-active metals such as copper and iron have been implicated in a variety of oxidative processes including $A \beta$ peptide-induced protein oxidation (20) and inactivation of putative antioxidant defenses such as heme oxygenase (21). Chelation of copper by the antibiotic iodochlorhydroxyquin (Clioquinol) has been found to effectively retard amyloid deposition in a mouse model of Alzheimer disease (22).

Genetic and biochemical manipulations to enhance antioxidant defenses provide sound support for the hypothesis that oxidative stress is a critical and common mechanism in neurodegeneration. Overexpression of $\mathrm{Cu} / \mathrm{Zn}-\mathrm{SOD}$ in transgenic mice and rats provides substantial protection against ischemia, cold edema, and neurotoxins and promotes survival of neurons in culture and after transplantation (23-25). Genetically induced increase in expression of Mn-SOD (26) or induction of the enzyme during stress (27) has been shown to protect mitochondria and cells from oxidative stress. Similarly, increased expression of extracellular SOD or glutathione peroxidase, as well as supplementation with SOD mimetics has been found to protect the CNS from a variety of neurotoxins $(28,29)$. In more simple models, overexpression of SOD and catalase significantly extended the lifespan of flies and worms (30-32). Remarkably, up to $60 \%$ of the lifespan of SOD knockout and catalase knockout Drosophila can be restored by expression of SOD in only the motor neurons (32). Overall, new evidence made possible by transgenic approaches implicates antioxidant enzymes and small antioxidants such as vitamins $\mathrm{E}$ and $\mathrm{C}$ and metal chelation in protecting the CNS.

\section{Sources of oxidants}

The generation of oxidants is often attributed to accidents of metabolism, with estimates that up to $1 \%$ of all oxygen consumption might be reduced to superoxide or hydrogen peroxide. Certainly, the use oxygen as the terminal electron acceptor poses a serious threat to cells because of the formation of partially reduced oxygen species. Generally, electron flow through oxygenutilizing processes such as the mitochondrial electrontransport chain, flavoproteins, cytochrome P450, and other oxidases is tightly coupled to avoid partial reduction of oxygen. Any potential interference with the electron transfer, such as the uncoupling of complex I of the mitochondrial electron transport and genetic defects of cytochrome oxidase, may contribute to neuronal degeneration $(33,34)$.

Oxidants can be produced by essentially all of the cells in the brain. For example, NADPH oxidase, the superoxide-generating enzyme in phagocytes, is expressed not only by microglia but also by astrocytes and neurons (35). However, neither superoxide nor hydrogen peroxide is particularly toxic (36). For toxicity to be observed 


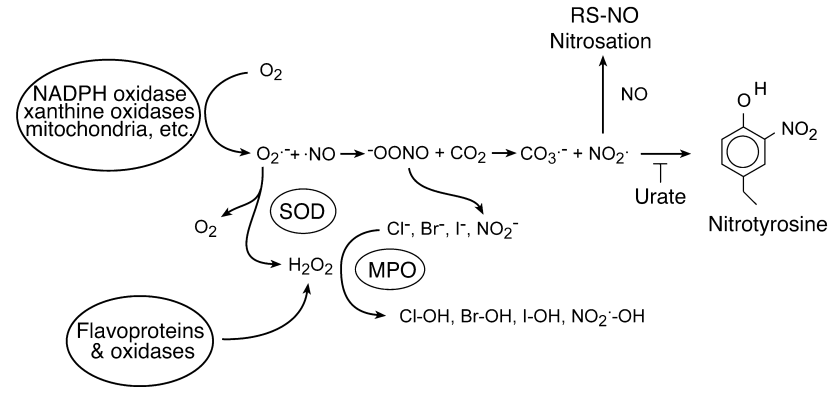

Figure 2

Common reactions of oxygen radicals and nitric oxide in a biological setting. NADPH oxidase and xanthine oxidase transfer a single electron to oxygen to form superoxide anion, while other flavoproteins can transfer two electrons to form hydrogen peroxide directly. SODs scavenge superoxide, which forms additional hydrogen peroxide. Peroxidases use hydrogen peroxide to oxidize a wide range of substrates to produce many different reactive species. Nitric oxide reacts with superoxide to form peroxynitrite. Carbon dioxide reacts catalytically with peroxynitrite to form nitrogen dioxide and carbonate radical. These two radicals nitrate tyrosine, which is blocked by the competitive inhibitor urate. Nitric oxide can also react with these radicals to form nitrosating intermediates that can produce, among other things, S-nitrosothiols. MPO, myeloperoxidase; RS-NO, S-nitrosothiols.

in most cell culture experiments, the concentration of hydrogen peroxide supplied must be more than the oxygen present in the media. Although hydroxyl radicals are commonly discussed in textbooks as the major toxic oxidant in vivo, their formation by the Haber-Weiss reaction or the Fenton reaction is too slow and too broadly reactive to be particularly toxic (36).

Alternatively, two distinct pathways are used to enhance the toxicity of partially reduced oxygen species. Inflammatory cells greatly enhance the toxicity of hydrogen peroxide by using peroxidases to produce hypochlorous acid ( $\mathrm{HOCl}$ ), better known as bleach, and other hypohalous acids like $\mathrm{HOBr}$ and $\mathrm{HOI}$ (Figure 2) (37). Cells can greatly increase the toxicity of superoxide by producing nitric oxide. These two radicals react to produce peroxynitrite $\left(\mathrm{ONOO}^{-}\right)$, by the fastest reaction known in biology and three times faster than previously thought (36). In the presence of carbon dioxide, peroxynitrite readily modifies proteins to form nitrotyrosine. Nitrotyrosine can be also formed by peroxidase oxidation of nitrite, a byproduct of nitric oxide metabolism, and hydrogen peroxide (38). The significance of nitric oxide contribution to neuronal injury is indicated by the use of nitric oxide synthase (NOS) inhibitors and is best documented by the use of mutant mice deficient in the neuronal isoform of NOS (NOS1) (39-43). Mice deficient in NOS1 were found to be resistant to stroke (40), $N$-methyl-D-aspartate neurotoxicity (41), and 1-methyl-4-phenyl-1,2,3,6-tetrahydropyridine (MPTP) toxicity (24). In addition to NOS1, other studies in human and animal models have also documented the contribution of NOS2, the inducible form of NOS, primarily found in brain glial cells (43, 44). For example, plaques in multiple sclerosis patients showed increased immunoreactivity for NOS2 and nitrotyrosine (6). Nitration has also been associated with compromised integrity of the blood-brain barrier in multiple sclerosis (45). In animal models of multiple sclerosis and stroke, uric acid has proved to be a useful inhibitor of tyrosine nitration and has been shown to protect the blood-brain barrier $(45,46)$. However, urate does not directly react with peroxynitrite and thus is not strictly a scavenger of peroxynitrite. Nitric oxide derived from NOS2 also contributes to neurotoxicity in ALS mouse models as well as in the MPTP model of Parkinson disease. Blockade of NOS2 and microglia activation by minocycline has been found to be neuroprotective in both Parkinson disease and ALS mouse models, offering hope for a potential therapeutic intervention $(47,48)$. Activation of microglia leading to peroxynitrite formation has also been linked to $A \beta$ peptide neurotoxicity (49).

\section{Oxidative and nitrative stress as a unifying mechanism in neurodegeneration}

Anatomical and histological studies have established the existence of selective regional vulnerability to neurodegeneration and cell death. For example, the dopaminergic neurons in the substantia nigra are selectively injured in Parkinson disease, whereas motor neurons in the spinal cord are selectively lost in ALS, and loss of cholinergic neurons frequently occurs in the forebrain of individuals with Alzheimer disease. Despite this regional sensitivity, oxidative processes may represent a specific and selective unifying mechanism for neurodegeneration. Several aspects of this working hypothesis are evident in Parkinson disease and ALS.

Parkinson disease. Parkinson disease may serve as an excellent example to discuss the significance of oxidative processes as a central but not an initiating event for the development of clinical disease (Figure 3). Environmental toxins (e.g., paraquat, rotenone, and MPTP) that epidemiological studies have shown to be risk factors are capable of generating reactive intermediates, directly alkylating reduced thiols, inhibiting complex I of the mitochondrial transport chain, inducing $\alpha$-synuclein aggregation, and activating microglia. Possibly, they may also alter iron or other divalent metal homeostasis as well as dopamine metabolism, permitting an increase in non-vesicle-associated dopamine levels. All these events permit formation of reactive oxygen and nitrogen intermediates that propagate cellular dysfunction, leading to cell death. Support for the working model depicted in Figure 3 comes from rodent and cellular models of Parkinson disease. Chronic administration of the mitochondrial electron-transport inhibitor rotenone was reported to recapitulate features of Parkinson disease in rats, including $\alpha$-synuclein inclusions (50). Rotenone was also found to induce $\alpha$-synuclein aggregation in cellular model systems of $\alpha$-synuclein overexpression $(51,52)$. Paraquat has been shown to upregulate and induce aggregation of $\alpha$-synuclein in mice (53) and in $\alpha$-synuclein-expressing cells (52).

The initiating event may also include genetic factors such as mutations of $\alpha$-synuclein. Mutations of $\alpha$-synuclein are responsible for familial Parkinson disease (54). 


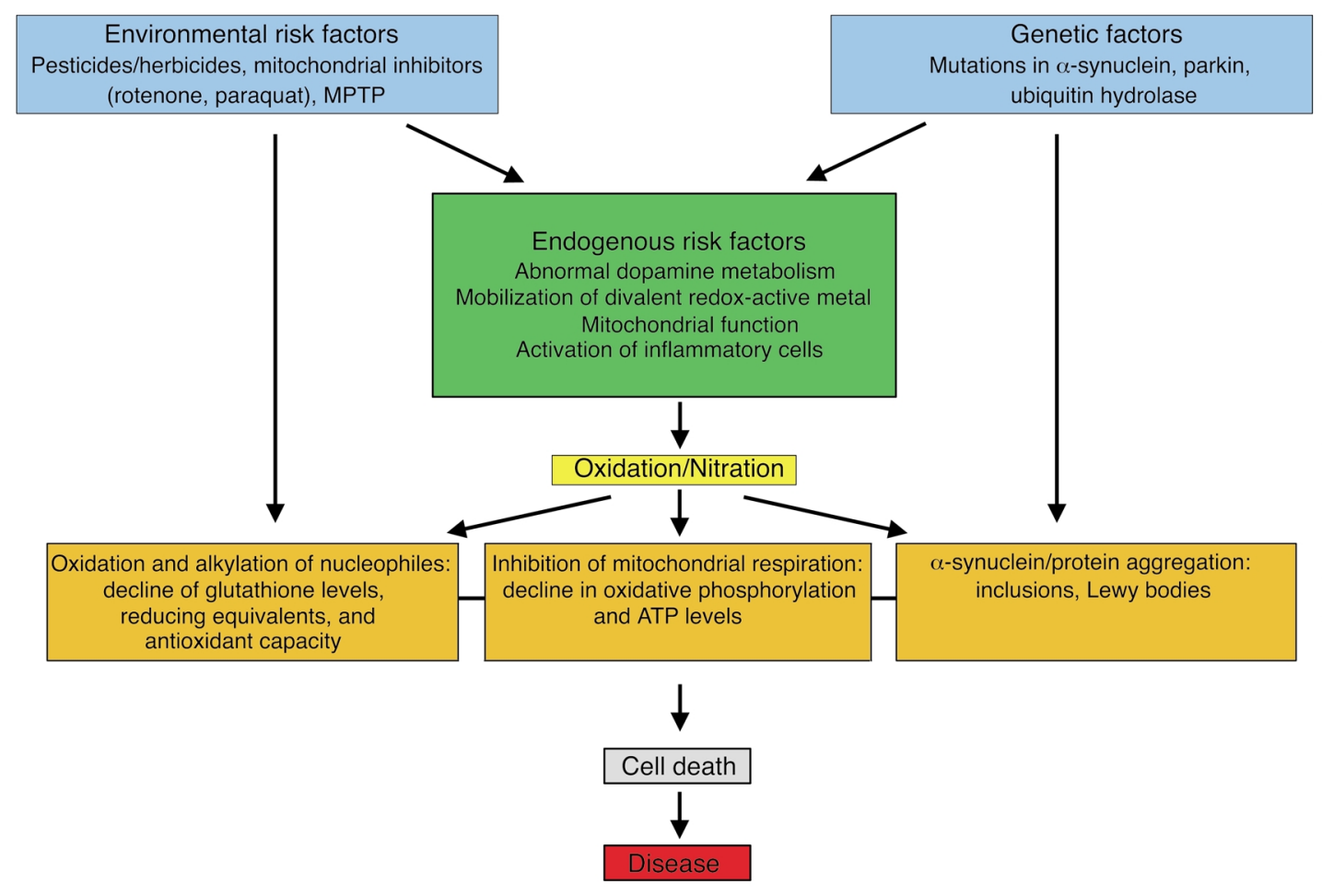

Figure 3

Proposed model for a central but not initiating role of oxidative processes in the pathogenesis of Parkinson disease. The model is based on published data and incorporates elements of the existing working hypothesis that places oxidative processes at the narrowest point of a funnel through which environmental, genetic, and endogenous risk factors flow to adversely impact cellular function and viability.

However, wild-type $\alpha$-synuclein appears to represent one of the major building blocks in Lewy bodies as well as other filamentous protein inclusions in sporadic Parkinson disease and several other pathologies (55). With the use of specific antibodies that recognize only the nitrated $\alpha$-synuclein, it is also apparent that the majority of the Lewy bodies and protein inclusions contain nitrated and possibly oxidized $\alpha$-synuclein, indicating that oxidative processes may participate in the formation of these inclusions (4) (Figure 1). Based on data from cellular model systems and in vitro biochemical studies, it is likely that oxidative and nitrative processes stabilize the formation of $\alpha$-synuclein aggregates in a manner that is resistant to proteolysis, thereby allowing the formation of highly insoluble protein aggregates (56-58). The selective nitration of $\alpha$-synuclein seen in protein inclusions indicates an unexpected biochemical event. Contrary to the belief that oxidants lack ability to select their biological targets, the reactivity is precisely described by the site of formation, as well as the concentration of reactive targets and the second-order rate constants between oxidant and targets. For example, tyrosine nitration is selective largely due to the local environment of certain tyrosine residues in proteins (59). The yield of nitration of these specific residues in selected proteins exceeds the yields typically achieved in proteins without reactive tyrosine residues or free tyrosine in the bulk solution (59). These observations imply that $\alpha$-synuclein is a primary and specific target for reactive nitrogen intermediates with the four tyrosine residues providing specific substrates for reactivity.

Support for the possibility that oxidative processes are the link between dopamine toxicity and $\alpha$-synuclein is provided by recent observations that expression of $\alpha$-synuclein predisposes cells to dopamine toxicity and that oxidants and nitrating agents generated by dopamine promote $\alpha$-synuclein aggregation $(52,60,61)$. The inclusion of dopamine in vesicles may represent a primary pathway for removing the potential toxicity of dopamine (62). In one cellular model, expression of $\alpha$-synuclein was found to disrupt the process of dopamine transport, increasing the steady-state levels of cytosolic dopamine sufficiently to promote cellular injury and apoptosis (63). The intracellular levels of dopamine may also be regulated by the direct interaction of $\alpha$-synuclein with tyrosine hydroxylase, the rate-limiting step in dopamine biosynthesis (64). Inhibition of tyrosine hydroxylase activity and a reduction in dopamine levels have been shown to inhibit $\alpha$-synuclein-induced apoptosis in cultured dopaminergic neurons (61). The toxicity of $\mathrm{MPP}^{+}$the active metabolite of MPTP, could be directly linked to the rise in intracellular non-vesicle-associated dopamine that leads to the generation of reactive intermediates (65). Furthermore, mice deficient in $\alpha$-synuclein have been found to have abnormal dopamine release following paired-pulse stimulation and to display a reduction in striatal dopamine, suggesting a possible interplay of $\alpha$-synuclein with synaptic 
function related to dopamine metabolism (66). It would be interesting to determine the responses of $\alpha$-synuclein knockout mice to neurotoxins such as MPTP, paraquat, and mitochondrial inhibitors and to compare and contrast them with the responses of $\alpha$-synuclein transgenic animals.

Dysregulation of dopamine transport should be considered a critical event in Parkinson disease. Free non-vesicle-associated dopamine is a source of reactive intermediates because of its potential to auto-oxidize to quinone generating reactive intermediates (62). Moreover, quinone addition on reduced sulfhydryls produces covalently linked protein adducts that can inactivate or alter protein function (62). Dopamine adducts with $\alpha$-synuclein have been recently shown to inhibit formation of $\alpha$-synuclein fibrils by promoting protofibril formation (67). In vitro studies support a toxic function of protofibrils but not of $\alpha$-synuclein fibrils, suggesting that oxidative processes may link dopamine and $\alpha$-synuclein toxicity (67). However, this assertion requires further documentation.

The significance of protein aggregation in neurodegenerative processes is unclear, but, for the most part, the process of protein aggregation and formation of inclusions that contain oxidized and nitrated $\alpha$-synuclein is not a function of aging but only a phenomenon that coincides with the clinical documentation of Parkinson disease and other related neurodegenerative disorders. The pathological significance of protein aggregation may relate to the effort of the cell to prevent or to attempt to repair the aggregates. It can be envisioned that the cellular ability to repair modified and aggregated protein is diminished due to a significant decline in ATP levels and reducing equivalents. Essentially, a double-hit hypothesis could be evoked, where inhibition of either mitochondrial respiration or protein aggregation alone is not sufficient to drive the cell to its demise but the combination of the two can indeed execute cell death. Overall, oxidative processes may operate in concert with other molecular events such as failure of energy production and respiration, ability to repair oxidized biomolecules and to sustain synaptic contacts and trophic support in the process of neurodegeneration. Therefore it is likely that antioxidant therapies are only partly protective, since they do not alleviate the pressure or burden of sustaining synaptic transmission and metabolic and trophic support that are equally important for cell survival.

$A L S$. The difficulties of establishing a causal connection between oxidative stress and neurodegeneration are perhaps best illustrated in ALS. ALS results from the progressive death of motor neurons, leading to rapid muscle degeneration and progressive paralysis. A decade ago, the first 13 of nearly a hundred different mutations were identified in the SOD1 locus encoding the cytosolic $\mathrm{Cu} / \mathrm{Zn}$-SOD (see www.alsod.org). Transgenic mice and rats expressing mutant SOD1 develop motor neuron disease, which clearly establishes a toxic gain of function in SOD (68).

These $\mathrm{Cu} / \mathrm{Zn}$-SOD mutations would appear to be a smoking gun for oxidative stress causing neurodegeneration. One can find lipid peroxidation and nitrotyrosine in both transgenic mice and human patients $(5,68)$. Yet, after a decade of research, the role of oxidants remains confusing, with many researchers now favoring protein aggregation over any causal involvement of oxidative stress in ALS (69). The mutations to $\mathrm{Cu} / \mathrm{Zn}$-SOD reveal how little we truly understand about the interplay of oxidants and antioxidant defenses like SOD in the brain. The antioxidant defenses against superoxide provided by SOD are formidable. In nontransgenic mice, SOD concentration is about $14 \mu \mathrm{M}$ (based on monomer size of 16,000 daltons) whereas oxygen concentrations are typically only $30 \mu \mathrm{M}$. In the transgenic mice expressing mutant $\mathrm{Cu} / \mathrm{Zn}-\mathrm{SOD}$, the concentrations of mutant protein are about $80 \mu \mathrm{M}$ in the "low-expressing" lines or nearly $6 \%$ of total protein. Enhancing any malfunction of such an abundant protein can have huge consequences.

We found that even the mutant $\mathrm{Cu} / \mathrm{Zn}$-SODs that cause the most rapid forms of ALS can form perfectly functional proteins with normal SOD activity (70). However, the mutant proteins are clearly less stable and more susceptible to losing their zinc and copper atoms. Because zinc is held about 7,000 times less tightly than copper, the mutations favor the accumulation of zincdeficient $\mathrm{Cu} / \mathrm{Zn}-\mathrm{SOD}$. The loss of zinc visibly changes the protein from green to blue $(70,71)$. The copper in zinc-deficient $\mathrm{Cu} / \mathrm{Zn}-\mathrm{SOD}$ is more accessible, and zincdeficient SOD rapidly oxidizes many intracellular antioxidants, like ascorbate and thiols. The copper in turn transfers these electrons to oxygen to produce superoxide, in effect allowing $\mathrm{Cu} / \mathrm{Zn}$-SOD to operate in reverse. With a low concentration of nitric oxide, zinc-deficient $\mathrm{Cu} / \mathrm{Zn}-\mathrm{SOD}$ catalyzes the generation of peroxynitrite while depleting cellular antioxidant defenses (71). Endogenous production of peroxynitrite is sufficient to activate apoptosis in motor neurons by activating caspases (72). Delivering zinc-deficient $\mathrm{Cu} / \mathrm{Zn}-\mathrm{SOD}$ to motor neurons is sufficient to cause motor neurons to die through a peroxynitrite-dependent mechanism (71). The zinc-deficient $\mathrm{Cu} / \mathrm{Zn}$-SOD hypothesis offers reasonable explanations for how $\mathrm{Cu} / \mathrm{Zn}-\mathrm{SOD}$ can be involved in ALS. Zinc-deficient wild-type $\mathrm{Cu} / \mathrm{Zn}$-SOD is just as toxic as zinc-deficient mutant $\mathrm{Cu} / \mathrm{Zn}-\mathrm{SOD}$, which explains how wild-type $\mathrm{Cu} / \mathrm{Zn}-\mathrm{SOD}$ could be involved in the vast majority of ALS cases without mutations in $\mathrm{Cu} / \mathrm{Zn}-\mathrm{SOD}$. The mutations only slightly weaken the affinity for zinc, and thus the potential for forming zinc-deficient $\mathrm{Cu} / \mathrm{Zn}-\mathrm{SOD}$ is increased.

However, some data in transgenic mice appear to argue against the zinc-deficient $\mathrm{Cu} / \mathrm{Zn}$-SOD hypothesis. Most recently, knocking out the gene for the copper chaperone for $\mathrm{Cu} / \mathrm{Zn}-\mathrm{SOD}$ (CCS) was found not to affect disease development in transgenic mice overexpressing mutant $\mathrm{Cu} / \mathrm{Zn}$-SODs (73). However, $15 \%$ of $\mathrm{Cu} / \mathrm{Zn}$-SOD activity remained in the CCS knockout mice, showing that the $\mathrm{Cu} / \mathrm{Zn}-\mathrm{SOD}$ could incorporate approximately $12 \mu \mathrm{M}$ copper from another cellular source possibly generating zinc-deficient $\mathrm{Cu} / \mathrm{Zn}-\mathrm{SOD}$. The zinc-deficient $\mathrm{Cu} / \mathrm{Zn}-\mathrm{SOD}$ hypothesis can explain the role of $\mathrm{Cu} / \mathrm{Zn}-\mathrm{SOD}$ in promoting the death of motor neurons, but not why 
the disease is progressive, causing the death of the few hundred thousand neurons that control all voluntary muscle contraction. Recently, peroxynitrite has been shown to provoke a long-lasting reactive phenotype in spinal cord astrocytes, and astrocytes have been shown to cause motor neurons in coculture to undergo apoptosis (74). In contrast, treatment of astrocytes with hydrogen peroxide does not induce apoptosis of cocultured motor neurons. A consistent finding in ALS spinal cord and in transgenic mice is an extremely strong immunoreactivity for nitrotyrosine associated with reactive astrocytes (5). By provoking surrounding astrocytes, a cluster of dying motor neurons may initiate a progressive death of neighboring motor neurons. Although the zincdeficient $\mathrm{Cu} / \mathrm{Zn}$-SOD hypothesis is highly controversial, it illustrates how much more subtle the role of oxidative and nitrative stress can be in neurodegeneration. Rather than causing overt necrotic death in motor neurons, oxidants can kill motor neurons by activating an apoptotic cascade far upstream. Instead of killing astrocytes, some oxidants induce a "proinflammatory" reactive phenotype that causes motor neurons to produce peroxynitrite and thus to activate apoptosis.

\section{Concluding comments and future directions}

Currently, there is sufficient documentation to place oxidative and nitrative processes in the center of the pathogenic mechanism that leads to neuronal loss and neurodegeneration. However, despite years of research efforts, the proximal sites of reactive-species generation remain elusive. A number of pathways have been identified as potential contributors, including the mitochondrial electron-transport chain, dopamine, membrane NADPH oxidases, and cytosolic flavoproteins. The most recent data have also identified nitric oxide-derived reactive nitrogen intermediates as critical contributors of protein modification and cell injury, providing potential targets for therapeutic interventions. Consideration should be also given to inappropriate regulation of iron and other divalent redox metals such as copper, as well as to redox-inactive zinc. Recent data have identified genetic defects that lead to iron accumulation in neurodegenerative diseases. Mutations in the gene that encodes the main iron-storage protein ferritin light polypeptide cause dominant adult-onset basal ganglia disease (75). Its presentation includes extrapyramidal features similar to those of Huntington disease and Parkinson disease with abnormal aggregation of ferritin and iron deposits. Abnormal iron accumulation and formation of $\alpha$-synuclein inclusions that prominently feature tyrosine-nitrated $\alpha$-synuclein are features of iron accumulation type 1 disease (76). This disease also presents with extrapyramidal dysfunction and a defect in the pantothenate kinase gene. As is mentioned above metals could drive the generation of strong oxidants and nitrating agents or directly be involved in the aggregation processes of proteins such as $\alpha$-synuclein, amyloid, ferritin, or other proteins.
Metal chelation strategies have proven effective in animal models of amyloid accumulation and are now being tested in clinical trials. On the other hand, binding of zinc to protein aggregates could potentially amplify disease by favoring the accumulation of zincdeficient SOD in ALS.

Overall oxidative processes play a critical role in the pathogenic mechanisms of neurodegenerative diseases. Promising therapeutic interventions targeted against oxidative processes could be explored in clinical trials to eventually relieve the burden of both acute and chronic neurodegeneration.

1. Lyras, L., et al. 1998. Oxidative damage to proteins, lipids, and DNA in cortical brain regions from patients with dementia with Lewy bodies. J. Neurochem. 71:302-312.

2. Hensley, K., et al. 1998. Electrochemical analysis of protein nitrotyrosine and dityrosine in the Alzheimer brain indicates region-specific accumulation. J. Neurosci. 18:8126-8132.

3. Pratico, D., et al. 2000. Increased 8,12-iso-iPF2alpha-VI in Alzheimer's disease: correlation of a noninvasive index of lipid peroxidation with disease severity. Ann. Neurol. 48:809-812.

4. Giasson, B.I., et al. 2000. Oxidative damage linked to neurodegeneration by selective alpha-synuclein nitration in synucleinopathy lesions. Science. 290:985-989.

5. Beal, M.F., et al. 1997. Increased 3-nitrotyrosine in both sporadic and familial amyotrophic lateral sclerosis. Ann. Neurol. 42:644-654.

6. Bagasra, O., et al. 1995. Activation of the inducible form of nitric oxide synthase in the brains of patients with multiple sclerosis. Proc. Natl. Acad. Sci. USA. 92:12041-12045.

7. Browne, S.E., Ferrante, R.J., and Beal, M.F. 1999. Oxidative stress in Huntington's disease. Brain Pathol. 9:147-163.

8. Moskovitz, J., et al. 2001. Methionine sulfoxide reductase (MsrA) is a regulator of antioxidant defense and lifespan in mammals. Proc. Natl. Acad. Sci. USA. 98:12920-12925.

9. Gabbita, S.P., Aksenov, M.Y., Lovell, M.A., and Markesbery, W.R. 1999. Decrease in peptide methionine sulfoxide reductase in Alzheimer's disease brain. J. Neurochem. 73:1660-1666.

10. De Boer, J., et al. 2002. Premature aging in mice deficient in DNA repair and transcription. Science. 296:1276-1279.

11. Troy, C.M., Derossi, D., Prochiantz, A., Greene, L.A., and Shelanski, M.L. 1996. Downregulation of $\mathrm{Cu} / \mathrm{Zn}$ superoxide dismutase leads to cell death via the nitric oxide-peroxynitrite pathway. J. Neurosci. 16:253-261.

12. Kondo, T., et al. 1997. Reduction of $\mathrm{Cu}, \mathrm{Zn}$-superoxide dismutase activity exacerbates neuronal cell injury and edema formation after transient focal cerebral ischemia. J. Neurosci. 17:4180-4189.

13. Lebovitz, R.M., et al. 1996. Neurodegeneration, myocardial injury, and perinatal death in mitochondrial superoxide dismutase-deficient mice. Proc. Natl. Acad. Sci. USA. 93:9782-9787.

14. Zhang, J., Graham, D.G., Montine, T.J., and Ho, Y.S. 2000. Enhanced $\mathrm{N}$-methyl-4-phenyl-1,2,3,6-tetrahydropyridine toxicity in mice deficient in $\mathrm{Cu}, \mathrm{Zn}$-superoxide dismutase or glutathione peroxidase. J. Neuropathol. Exp. Neurol. 59:53-61.

15. Yokota, T., et al. 2001. Delayed-onset ataxia in mice lacking alpha-tocopherol transfer protein: model for neuronal degeneration caused by chronic oxidative stress. Proc. Natl. Acad. Sci. USA. 98:15185-15190.

16. Sano, M., et al. 1997. A controlled trial of selegiline, alpha-tocopherol, or both as treatment for Alzheimer's disease. The Alzheimer's Disease Cooperative Study. N. Engl. J. Med. 336:1216-1222.

17. Desnuelle, C., Dib, M., Garrel, C., and Favier, A. 2001. A double-blind, placebo-controlled randomized clinical trial of alpha-tocopherol (vita$\mathrm{min} \mathrm{E}$ ) in the treatment of amyotrophic lateral sclerosis. ALS riluzoletocopherol Study Group. Amyotroph. Lateral Scler. Other Motor Neuron Disord. 2:9-18.

18. Huang, J., et al. 2001. Dehydroascorbic acid, a blood-brain barrier transportable form of vitamin C, mediates potent cerebroprotection in experimental stroke. Proc. Natl. Acad. Sci. USA. 98:11720-11724.

19. Sotiriou, S., et al. 2002. Ascorbic-acid transporter Slc23a1 is essential for vitamin $\mathrm{C}$ transport into brain and for perinatal survival. Nat. Med. 8:514-517.

20. Huang, X., et al. 1999. Cu(II) potentiation of Alzheimer Abeta neurotoxicity. Correlation with cell-free hydrogen peroxide production and metal reduction. J. Biol. Chem. 274:37111-37116.

21. Takahashi, M., et al. 2000. Amyloid precursor proteins inhibit heme oxygenase activity and augment neurotoxicity in Alzheimer's disease. Neuron. 28:461-473.

22. Cherny, R.A., et al. 2001. Treatment with a copper-zinc chelator markedly 
and rapidly inhibits beta-amyloid accumulation in Alzheimer's disease transgenic mice. Neuron. 30:665-676.

23. Chan, P.H., Yang, G.Y., Chen, S.F., Carlson, E., and Epstein, C.J. 1991 Cold-induced brain edema and infarction are reduced in transgenic mice overexpressing Cu, Zn-superoxide dismutase. Ann. Neurol. 29:482-486.

24. Przedborski, S., et al. 1992. Transgenic mice with increased $\mathrm{Cu} / \mathrm{Zn}$-superoxide dismutase activity are resistant to N-methyl-4-phenyl-1,2,3,6tetrahydropyridine-induced neurotoxicity. J. Neurosci. 12:1658-1667.

25. Nakao, N., et al. 1995. Overexpressing $\mathrm{Cu} / \mathrm{Zn}$ superoxide dismutase enhances survival of transplanted neurons in a rat model of Parkinson's disease. Nat. Med. 1:226-231.

26. Keller, J.N., et al. 1998. Mitochondrial manganese superoxide dismutase prevents neural apoptosis and reduces ischemic brain injury: suppression of peroxynitrite production, lipid peroxidation, and mitochondrial dysfunction. J. Neurosci. 18:687-697.

27. Dawson, V.L., Kizushi, V.M., Huang, P.L., Snyder, S.H., and Dawson, T.M. 1996. Resistance to neurotoxicity in cortical cultures from neuronal nitric oxide synthase-deficient mice. J. Neurosci. 16:2479-2487.

28. Pineda, J.A., et al. 2001. Extracellular superoxide dismutase overexpression improves behavioral outcome from closed head injury in the mouse. J. Neurotrauma. 18:625-634.

29. Pong, K., Doctrow, S.R., and Baudry, M. 2000. Prevention of 1-methyl-4phenylpyridinium- and 6-hydroxydopamine-induced nitration of tyrosine hydroxylase and neurotoxicity by EUK-134, a superoxide dismutase and catalase mimetic, in cultured dopaminergic neurons. Brain Res. 881:182-189.

30. Orr, W.C., and Sohal, R.S. 1994. Extension of life-span by overexpression of superoxide dismutase and catalase in Drosophila melanogaster. Science. 263:1128-1130.

31. Melov, S., et al. 2000. Extension of life-span with superoxide dismutase/catalase mimetics. Science. 289:1567-1569.

32. Parkes, T.L., et al. 1998. Extension of Drosophila lifespan by overexpression of human SOD1 in motor neurons. Nat. Genet. 19:171-174.

33. Swerdlow, R.H., et al. 1996. Origin and functional consequences of the complex I defect in Parkinson's disease. Ann. Neurol. 40:663-671.

34. Davis, R.E., et al. 1997. Mutations in mitochondrial cytochrome c oxidase genes segregate with late-onset Alzheimer disease. Proc. Natl. Acad. Sci. USA. 94:4526-4531.

35. Tammariello, S.P., Quinn, M.T., and Estus, S. 2000. NADPH oxidase contributes directly to oxidative stress and apoptosis in nerve growth factordeprived sympathetic neurons. J. Neurosci. 20:RC53.

36. Beckman, J.S. 1994. Peroxynitrite versus hydroxyl radical: the role of nitric oxide in superoxide-dependent cerebral injury. In The neurobiology of NO_and_OH. C.C.Chiueh, D.L. Gilbert, and C.A. Colton, editors. New York Academy of Sciences. New York, New York, USA. 69-75.

37. Harrison, J.E., and Schultz, J. 1976. Studies on the chlorinating activity of myeloperoxidase. J. Biol. Chem. 251:1371-1374.

38. Brennan, M.L., et al. 2002. A tale of two controversies: defining both the role of peroxidases in nitrotyrosine formation in vivo using eosinophil peroxidase and meyloperoxidase-deficient mice, and the nature of peroxidasegenerated reactive nitrogen species. J. Biol. Chem. 277:17415-17427.

39. Hantraye, P., et al. 1996. Inhibition of neuronal nitric oxide synthase prevents MPTP-induced parkinsonism in baboons. Nat. Med. 2:1017-1021.

40. Eliasson, M.J., et al. 1999. Neuronal nitric oxide synthase activation and peroxynitrite formation in ischemic stroke linked to neural damage. J. Neurosci. 19:5910-5918.

41. Ayata, C., et al. 1997. Mechanisms of reduced striatal NMDA excitotoxicity in type I nitric oxide synthase knock-out mice. J. Neurosci. 17:6908-6917.

42. Przedborski, S., et al. 1996. Role of neuronal nitric oxide in 1-methyl-4phenyl-1,2,3,6-tetrahydropyridine (MPTP)-induced dopaminergic neurotoxicity. Proc. Natl. Acad. Sci. USA. 93:4565-4571.

43. Liberatore, G.T., et al. 1999. Inducible nitric oxide synthase stimulates dopaminergic neurodegeneration in the MPTP model of Parkinson disease. Nat. Med. 5:1403-1409.

44. Vodovotz, Y., et al. 1996. Inducible nitric oxide synthase in tangle-bearing neurons of patients with Alzheimer's disease. J. Exp. Med. 184:1425-1433.

45. Kean, R.B., Spitsin, S.V., Mikheeva, T., Scott, G.S., and Hooper, D.C. 2000. The peroxynitrite scavenger uric acid prevents inflammatory cell invasion into central nervous system in experimental allergic encephalomyelitis through maintenance of blood-central nervous system barrier integrity. J. Immunol. 165:6511-6518.

46. Yu, Z.F., Bruce-Keller, A.J., Goodman, Y., and Mattson, M.P. 1998. Uric acid protects neurons against excitotoxic and metabolic insults in cell culture, and against focal ischemic brain injury in vivo. J. Neurosci. Res. 53:613-625.

47. Wu, D.C., et al. 2002. Blockade of microglial activation is neuroprotective in the 1-methyl-4-phenyl-1,2,3,6-tetrahydropyridine mouse model of Parkinson disease. J. Neurosci. 22:1763-1771.
48. Zhu, S., et al. 2002. Minocycline inhibits cytochrome c release and delays progression of amyotrophic lateral sclerosis in mice. Nature. 417:74-78.

49. Xie, Z., et al. 2002. Peroxynitrite mediates neurotoxicity of amyloid betapeptide1-42- and lipopolysaccharide-activated microglia. J. Neurosci. 22:3484-3492.

50. Betarbet, R., et al. 2000. Chronic systemic pesticide exposure reproduces features of Parkinson's disease. Nat. Neurosci. 3:1301-1306

51. Manning-Bog, A.B., et al. 2002. The herbicide paraquat causes up-regulation and aggregation of alpha-synuclein in mice: paraquat and alphasynuclein. J. Biol. Chem. 277:1641-1644.

52. Lee, H.J., Shin, S.Y., Choi, C., Lee, Y.H., and Lee, S.J. 2002. Formation and removal of alpha-synuclein aggregates in cells exposed to mitochondrial inhibitors. J. Biol. Chem. 277:5411-5417.

53. Paxinou, E., et al. 2001. Induction of alpha-synuclein aggregation by intracellular nitrative insult. J. Neurosci. 21:8053-8061.

54. Polymeropoulos, M.H., et al. 1997. Mutation in the alpha-synuclein gene identified in families with Parkinson's disease. Science. 276:2045-2047.

55. Spillantini, M.G., et al. 1997. Alpha-synuclein in Lewy bodies. Nature. 388:839-840.

56. Hashimoto, M., et al. 1999. Oxidative stress induces amyloid-like aggregate formation of NACP/alpha- synuclein in vitro. Neuroreport. 10:717-721.

57. Ostrerova-Golts, N., et al. 2000. The A53T alpha-synuclein mutation increases iron-dependent aggregation and toxicity. J. Neurosci. 20:6048-6054.

58. Souza, J.M., Giasson, B.I., Chen, Q., Lee, V.M., and Ischiropoulos, H. 2000. Dityrosine cross-linking promotes formation of stable alpha-synuclein polymers. Implication of nitrative and oxidative stress in the pathogenesis of neurodegenerative synucleinopathies. J. Biol. Chem. 275:18344-18349.

59. Souza, J.M., Daikhin, E., Yudkoff, M., Raman, C.S., and Ischiropoulos, H. 1999. Factors determining the selectivity of protein tyrosine nitration. Arch. Biochem. Biophys. 371:169-178.

60. Tabrizi, S.J., et al. 2000. Expression of mutant alpha-synuclein causes increased susceptibility to dopamine toxicity. Hum. Mol. Genet. 9:2683-2689.

61. Xu, J., et al. 2002. Dopamine-dependent neurotoxicity of alpha-synuclein: a mechanism for selective neurodegeneration in Parkinson disease. Nat. Med. 8:600-606.

62. Hastings, T.G., Lewis, D.A., and Zigmond, M.J. 1996. Role of oxidation in the neurotoxic effects of intrastriatal dopamine injections. Proc. Natl. Acad. Sci. USA. 93:1956-1961.

63. Lee, F.J.S., Liu, F., Pristupa, Z.B., and Niznik, H.B. 2001. Direct binding and functional coupling of a-synuclein to the dopamine transporters accelerate dopamine-induced apoptosis. FASEB J. 15:916-926.

64. Perez, R.G., et al. 2002. A role for a-synuclein in the regulation of dopamine biosynthesis. J. Neurosci. 22:3090-3099.

65. Lotharius, J., and O'Malley, K.L. 2000. The parkinsonism-inducing drug 1-methyl-4-phenylpyridinium triggers intracellular dopamine oxidation. A novel mechanism of toxicity. J. Biol. Chem. 275:38581-38588.

66. Abeliovich, A., et al. 2000. Mice lacking alpha-synuclein display functional deficits in the nigrostriatal dopamine system. Neuron. 25:239-252.

67. Conway, K.A., Rochet, J.C., Bieganski, R.M., and Lansbury, P.T., Jr. 2001 Kinetic stabilization of the alpha-synuclein protofibril by a dopaminealpha-synuclein adduct. Science. 294:1346-1349.

68. Gurney, M.E., et al. 1994. Motor neuron degeneration in mice that express a human $\mathrm{Cu}, \mathrm{Zn}$ superoxide dismutase mutation. Science. 264:1772-1775

69. Williamson, T.L., et al. 2000. Toxicity of ALS-linked SOD1 mutants. Science. 288:399.

70. Crow, J.P., et al. 1997. Decreased zinc affinity of amyotrophic lateral sclerosis-associated superoxide dismutase mutants leads to enhanced catalysis of tyrosine nitration by peroxynitrite. J. Neurochem. 69:1936-1944.

71. Estévez, A.G., et al. 1999. Induction of nitric oxide-dependent apoptosis in motor neurons by zinc-deficient superoxide dismutase. Science. 286:2498-2500

72. Estévez, A.G., et al. 1998. Nitric oxide and superoxide contribute to motor neuron apoptosis induced by trophic factor deprivation. J. Neurosci. 18:923-931.

73. Subramaniam, J.R., et al. 2002. Mutant SOD1 causes motor neuron disease independent of copper chaperone-mediated copper loading. Nat. Neurosci. 5:301-307.

74. Cassina, P., et al. 2002. Peroxynitrite triggers a phenotypic transformation in spinal cord astrocytes that induces motor neuron apoptosis. J. Neurosci. Res. 67:21-29.

75. Curtis, A.R., et al. 2001. Mutation in the gene encoding ferritin light polypeptide causes dominant adult-onset basal ganglia disease. Nat. Genet. 28:350-354.

76. Zhou, B., et al. 2001. A novel pantothenate kinase gene (PANK2) is defective in Hallervorden-Spatz syndrome. Nat. Genet. 28:345-349. 